\title{
Multipath ring Routing in Wireless Sensor Networks
}

\author{
Guimin Huang \\ Research Center on Data Science and Social Computing \\ Guilin University of Electronic Technology \\ Guilin, China \\ sendhuang@126.com
}

\author{
Wujin Tao, Pingshan Liu, Siyun Liu \\ Research Center on Data Science and Social Computing \\ Guilin University of Electronic Technology \\ Guilin, China \\ taowujin@163.com, liup3@guet.edu.cn, \\ lsiyun@qq.com
}

\begin{abstract}
In the past years, the routing problems of wireless sensor network (WSN) have attracted great interest, and many routing protocols for WSN have being proposed. However, these routing protocols pay little attention to the transmission reliability. Therefore, this paper proposes a new multipath ring routing algorithm for WSNs. The proposed algorithm utilizes ring level to separate sensor nodes into several sections, which can improve the reliability of the data transmission. Using the multipath ring routing data delivery improves as an average of $27.58 \%$ to leach routing and $113.06 \%$ to directing routing.
\end{abstract}

Keywords-multipath routing; transmission reliability; wireless sensor network

\section{INTRODUCTION}

A wireless sensor network (WSN) which consists of light-weight, low-power, computation-limited small size sensor nodes monitor physical or environmental conditions, such as temperature, sound, and pressure, etc. WSN is a spatially distributed, densely deployed and selforganized network [2], which is widely used in many domains, e.g. military situation awareness [3], battlefield surveillance [4], fire detectors, warning systems [5], machine health monitoring and so on. One of the most important design and implementation rules of a typical sensor network is reliability. While a node sends a packet to the sink node, the node may lose the packet during the routing process due to environmental effects, interference between nodes, instable link or other reasons. Therefore, redesigning a robust routing algorithm to ensure correctness of data transmission is vital to the WSNs.

This paper proposes a new multipath ring routing algorithm for WSNs, which shows a better performance than others in transmission reliability. The rest of the paper is organized as follows. In section 2, we provide related works. Then, we discuss route establishment of the multipath ring routing in section 3 . In section 4 , we evaluate the proposed algorithm through simulations. Last, we conclude the paper and discuss future works in section 5.

\section{REALTED WORKS}

In the past years, the routing problems of WSN have attracted great interest, and many routing protocols for WSN have being proposed, e.g. cluster-based routing, multipath routing and energy aware routing, which have been widely utilized for various network purposes, such as improving data delivery reliability, providing fault-tolerant routing, congestion control, machine health monitoring, Quality of Service (QoS) support and so on [6]. The author of [7] proposed an energy-efficient adaptive multipath routing technique which intends to make use of the residual energy and received signal strength to discover multiple paths to the sink node by reliable data transmission and low energy consumption. The authors in [8] proposed a self-optimized multipath routing protocol which utilizes the ant colony optimization that has shown excellent results in discovering routes for WSNs. In [9], the author presents a dynamic multipath routing algorithm in which packets from different applications dynamically choose their own paths by estimating the power to be consumed for each path and the ability to pay.

As we know, multipath routing algorithm provides multiple paths to the destination, but the interference will increase with the paths. However, an interferenceminimized multipath routing with congestion control in WSN for high-rate streaming was proposed in paper [10]. WSNs are often deployed for collecting information from unattended or hostile environment and paid little attention to security issues. In paper [11], the authors explored the general security problems in WSN and analyzed possible security problems on gathering algorithms.

\section{MULTIPATH RING ROUTING SCHEME}

\section{A. Multipath Construction Phase}

The basic idea of multipath construction phase is to organize the network into levels according to hop distance from the sink node to a sensor node i.e. by the end of this phase each node will get a ring level which indicates how many hops away from the sink node [1] [7]. In this phase, the sink node broadcasts a packet with its ring level 0 . The nodes which received the packet will increase their ring number and rebroadcast the packet to their neighbors. In the end, all the nodes in WSN are separated into several levels.

The format of Multiple Route Topology setup packet is shown in Fig. 1. The Source node ID is the ID of the node which broadcasts the setup packet. Destination node ID contains the node ID of the packet destination; the Sink node ID indicates the ID of the sink which is useful when 
a network is dealing with multiple sink nodes. The Packet type field is a flag to define packet type, which contains Multiple Route Topology setup packet and multipath ring routing data packet. The SeqNumber field provides a packet sequence, the Ring level is the number of hops from the sink node which is used to indicate nodes in different levels.

During multipath construction phase, the main work is ring level generation for all nodes. The sink node broadcasts the topology setup packet, which is used to explore the neighbor nodes that are receiving it first. We defined these nodes as level 1 node. Topology setup packet is used to identify nodes in different levels. When received a topology setup packet, a node considers itself in level $\mathrm{n}$ if the hop count is $\mathrm{n}$. If a smaller ring level is received later, then the node's level updates according to the new ring level.

\begin{tabular}{|l|l|l|l|l|l|}
\hline $\begin{array}{l}\text { Source } \\
\text { node ID }\end{array}$ & $\begin{array}{l}\text { Destination } \\
\text { node ID }\end{array}$ & $\begin{array}{l}\text { Sink } \\
\text { node ID }\end{array}$ & $\begin{array}{l}\text { Packet } \\
\text { type }\end{array}$ & SeqNumber & $\begin{array}{l}\text { Ring } \\
\text { level }\end{array}$ \\
\hline
\end{tabular}

Figure 1. Multiple Route Topology setup packet format

Fig. 2 shows the action flow diagram of multipath construction when the node receives the topology setup packet. Once the topology setup packet that send by the sink node arrived at a sensor node, the corresponding node computes the signal strength and decides whether to receive it or not. If the received signal strength is stronger than the receiver sensitivity, the packet is received and processed or otherwise drop it. After received the topology setup packet, we then have to make sure whether the current node's ring level is equal to -1or not. If current node's ring level is -1 , one should make it to be the received ring level plus one, then the node is responsible to rebroadcast the topology setup packet with the current ring level to its neighbors. If current node's ring level is not -1 , one should compare it with the received ring level. While the received ring level plus one is smaller than the current node's ring level, one should update the node's ring level and set it to be the received ring level plus one, otherwise drop it. The nodes which rebroadcast the topology setup packet are available to provide a path to the destination. Unlike many other power-aware routing algorithms, which focus on finding minimum energy cost paths [9] [10], this algorithm attempt to adopt all energysufficient paths instead.

Before the construction of the multipath ring routing network, the sensor nodes are deployed in a random fashion or in a pre-arranged way [7]. As shown in fig. 3, initially the sink node marked " 0 " is with a ring level of " 0 " and all other nodes are with a ring level of " -1 ". While the sink node broadcast the topology setup packet which will be received by the nodes within its radio range. The nodes have received the packet will set their ring level to " 1 ", same as these nodes which also rebroadcast the topology setup packet to their neighbors. This process will not stop until all the nodes get their own ring level.

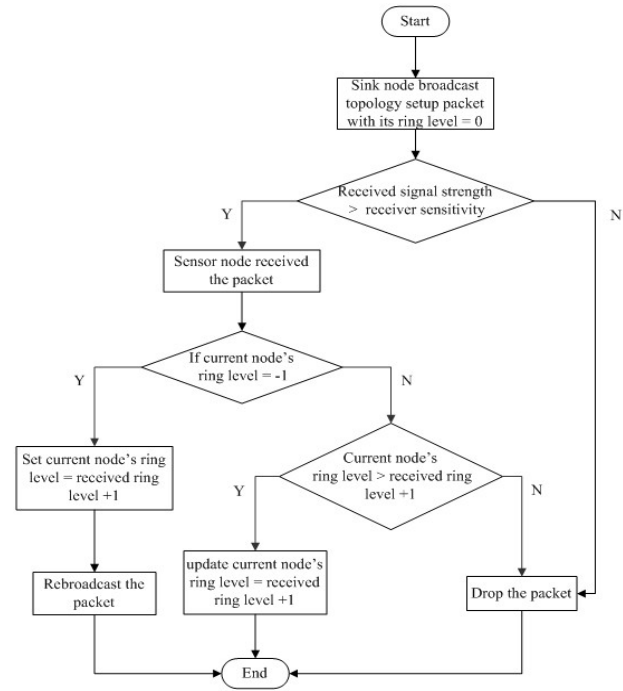

Figure 2. Flow chart of multipath construction

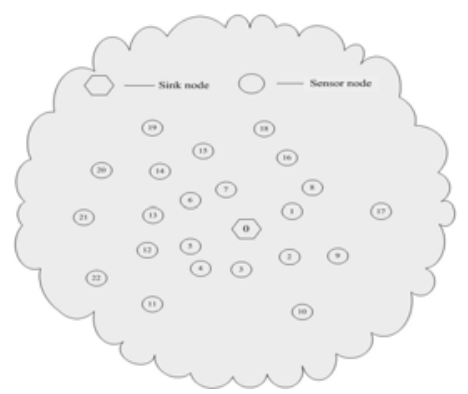

Figure 3. Sensor nodes before multipath construction

The Fig. 4 shows the details of the construction of the multipath ring routing network. In the begin, the sink node 0 broadcast the topology setup packet, the nodes $1,2,3,4$, 5, 6 and 7 receive the packet and update their ring level to 1. Assume that these nodes have enough energy, and then they rebroadcast the topology setup packet with the new ring level " 1 " to all their neighbors, such as nodes $8,9,12$ and so on. In the end all sensor nodes have got a new ring level which is the key issue for this routing protocol. After completing the ring level allocation among the sensor nodes, each node get a ring level which is stored in the node itself. From the Fig. 5 we can know which level is the node locates clearly, which is also showed in Tab. 1

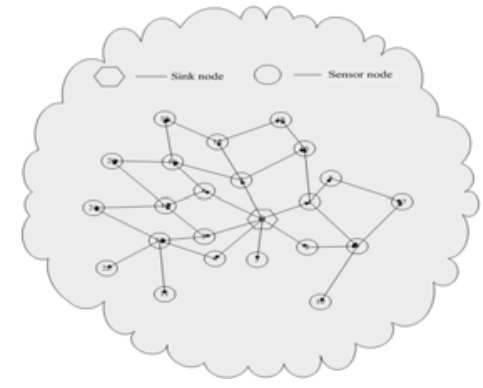

Figure 4. Sensor nodes after multipath construction 
TABLE I. LEVEL TABLE

\begin{tabular}{|c|c|}
\hline Ring level & \multicolumn{1}{|c|}{ Node ID } \\
\hline 0 & 0 (sink node ) \\
\hline 1 & 1234567 \\
\hline 2 & 891213141516 \\
\hline 3 & 1011171819202122 \\
\hline
\end{tabular}

In this routing algorithm, we do not need routing tables. As shown in Fig. 6 we know that the sink node is in the top of the tower and sensor nodes are in other levels. When we send a packet to the sink node, we just broadcast it and only the lower levels can receive and rebroadcast it until it arrive at the sink node.

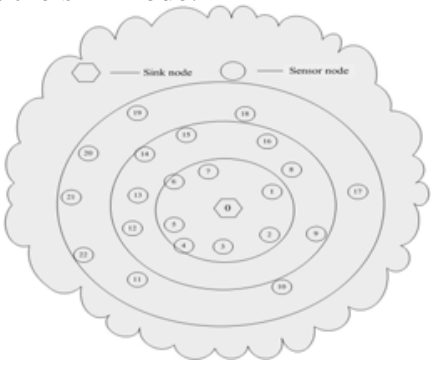

Figure 5. Sensor nodes in multipath ring routing network

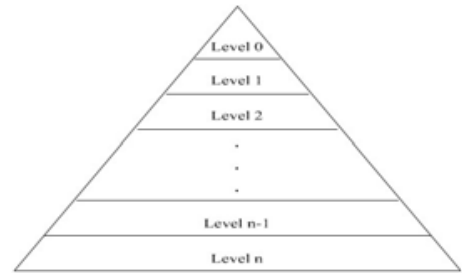

Figure 6. Tower level of the sensor nodes

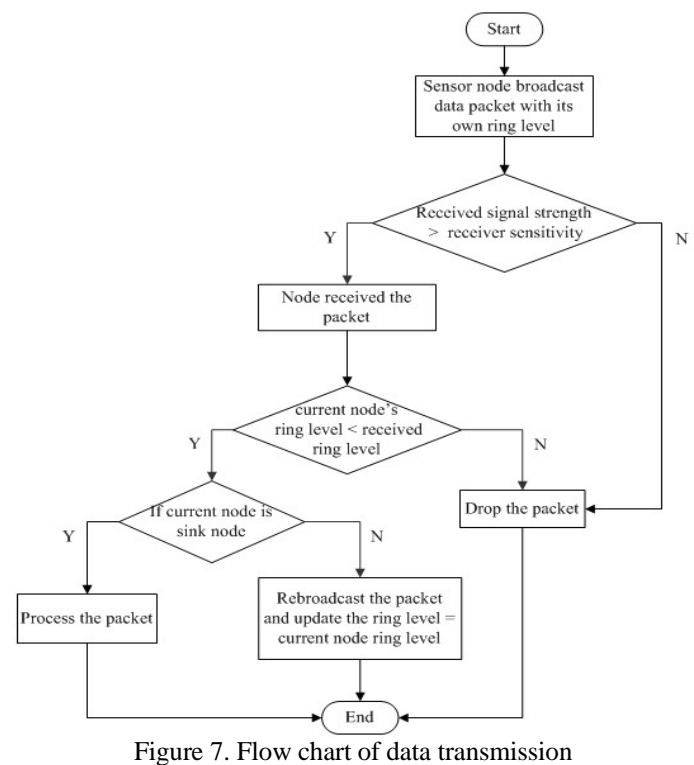

\section{B. Data Ttransmission Phase}

Now each sensor node has got its own ring level, then they can send data packets to the sink node. Due to the lack of address in wireless sensor network, we can not send a packet to the sink node directly but broadcast it with the node's ring level. If the nodes' ring level is lower than the received packet's ring level, then broadcast the packet with current node's ring level again, till the packet arrived at the sink node. The flow chart of data transmission is shown in fig. 7. From it, we know that if a sensor node wants to send a data to the sink node, it should broadcast the data with its own ring level. The neighbors around the node will receive the data and compare the received ring level with their own ring level, only the lower nodes can process the data. If the received node is the sink node then process the packet or otherwise rebroadcast the data and update the ring level.

In Fig. 8, we show an example of how data transmit from the sensor nodes to the sink node. As fig. 8 shows, node 18 and node 24 both send a sensor data to the sink node. First of all, the two nodes broadcast their data with ring level 3, and then only the lower level nodes can receive and process the data which are nodes $12,13,15$, 16. When these nodes receive the data they also broadcast it to their neighbors with ring level 2. Just like the last one, only these nodes in lower level will receive the data and process it. You may have known that, nodes 1, 4, 5, 6, 7 will receive the data and send it to the sink node with ring level 1. At last, the data arrived at the sink node and then send it to the application layer for further process. During the transmission we can see that, there are many paths to send a data to the sink node which robust the data transmission and decrease the error rate.

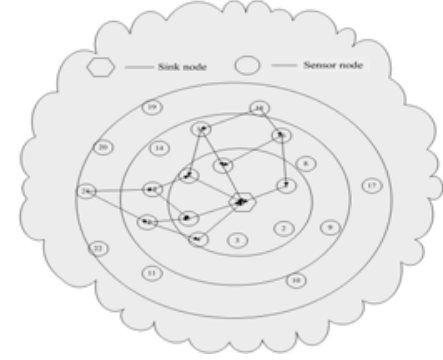

Figure 8. Data transmission in multipath ring routing network (1)

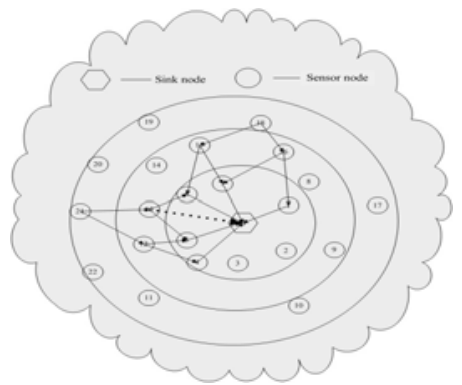

Figure 9. Data transmission in multipath ring routing network (2)

As we know, the signal strength has a lot to do with the environment. When a node in a bad day, it may not receive the packet from the sink node, while in a sunny day it can receive the packet successfully. As the example shown in Fig. 9, when the environment is bad enough, node 13 would not receive the topology setup packet from the sink node, then its ring level will be 2 , while the 
environment get better node 13 may send a data to the sink node directly rather than the nodes within ring level 1.

\section{EXPERIMENTS AND ANALYSIS}

To evaluate the multipath ring routing protocol, we use the OMNet++ based simulator Castalia, which is a simulator for WSN, Body Area Networks (BAN) and generally networks of low-power embedded devices.

In our experiments, we simulate the multipath ring routing algorithm with 6 groups' different numbers of nodes and compare it with direct routing and leach routing. Direct routing is one of the flooding protocols which just rebroadcast the received packet simply, while the leach routing is a cluster based algorithm. The simulation results are showed in Fig. 10 and Fig. 11.

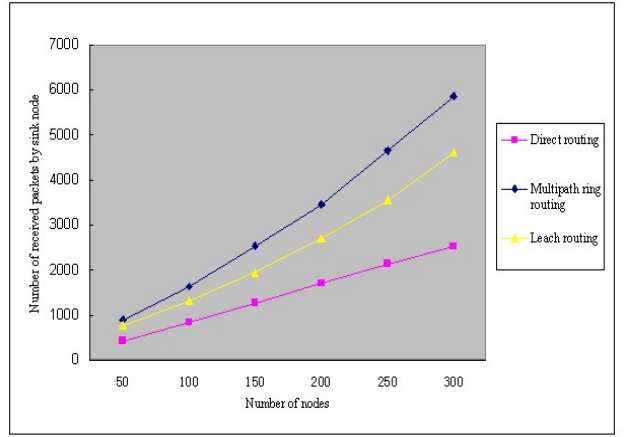

Figure 10. Comparison between different routing algorithms

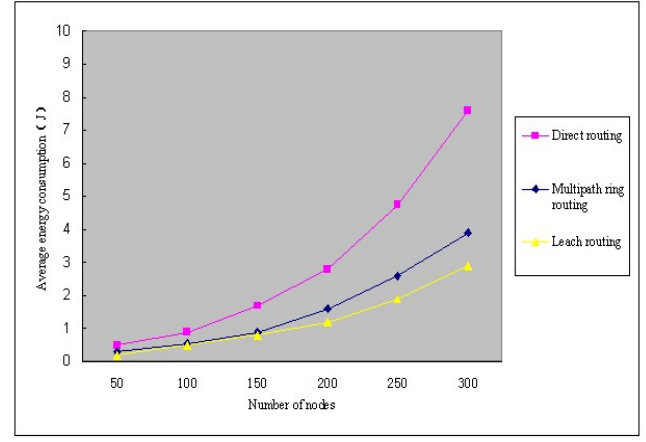

Figure 11. Average energy consumption

From Fig. 10, we know that multipath ring routing has the lowest packet loss when compared with other two routing algorithms. The direct routing gets the highest packet loss due to the lack of routing algorithm to delivery data to the sink node. Using the multipath ring routing data delivery improves as an average of $27.58 \%$ to leach routing and $113.06 \%$ to directing routing.

In Fig. 11 we compute the energy consumption for these routing algorithms, from which we can get a conclusion that leach routing algorithm has the least energy consumption compare with other two routing algorithms and the direct routing has the highest energy consumption. That is due to many nodes send their packets to the cluster head in a cluster which save much energy in leach routing algorithm while the direct routing is just broadcasting the received packets.

\section{CONCLUSION AND FUTUREWORKS}

In this paper, we have proposed an advanced multipath routing algorithm for WSNs. Compared with the other two routing algorithms which are direct routing and leach routing for WSNs, our proposed algorithm can improve the reliability of data transmission obviously. However, there is another question about the energy consumption for our proposed algorithm. Much research work has demonstrated that the cluster based routing algorithms consumed less energy than the multipath routing algorithms. Therefore, we will make an extensive study about the energy consumption for the routing algorithms in the near future.

\section{ACKNOWLEDGMENT}

This work is supported by the research Foundation of Key Laboratory of Wireless Broadband Communication and Signal Processing, No.11108.

\section{REFERENCES}

[1] Anand Pandya, Mrudang Mehta, "Performance Evaluation of Multipath Ring Routing Protocol for Wireless Sensor Network," UACEE International Journal of Advances in Computer Networks and its Security, vol. 2, no. 2, pp. 53-58, August 2012.

[2] Chunting Liu, Hong Huo, Tao Fang, Deren, Li, Xiao Shen, "Classification Fusion in Wireless Sensor Networks," ACTA AUTOMATIC ASINICA, vol. 32, no. 6, pp. 947-955, November 2006.

[3] Shen, C.C, Srisathapornphat, C, Jaikaeo, C. "Sensor Information Networking Architecture and Applications,” IEEE Personal Communications, vol. 8, no. 4, pp. 52-59, August 2001.

[4] Elaine Shi, Adrian Perrig, "Designing Secure Sensor Networks," IEEE Wireless Communications, vol. 11, no.6, pp. 38-43, December 2004

[5] Sarjoun S. Doumit, Dharma p. Agrawal, "Self-organizing and energy-efficient network of sensors," Proceedings Military Communications Conference 2002 (MILCOM 2002), vol. 2, pp. 1245-1250, October 2002.

[6] Marjan Radi, Behnam Dezfouli, Kamalrulnizam Abu Bakar, Malrey Lee, "Multipath Routing in Wireless Sensor Networks: Survey and Research Challenges,” Sensors, 12(1), pp. 650-685. January 2012.

[7] R Vidhyapriya, Dr P T Vanathi, "Energy Efficient Adaptive Multipath Routing for Wireless Sensor Networks," IAENG International Journal of Computer Science, 34(1), 2007.

[8] K. Saleem, N. Fisal, S. Hafizah, S. Kamilah, R. A. Rashid, “A Self-Optimized Multipath Routing Protocol for Wireless Sensor Networks,” International Journal of Recent Trends in Engineering, vol. 2, no. 1, pp. 93-97, November 2009.

[9] Syed Yousaf Shah and Boleslaw K. Szymanski, "Dynamic Multipath Routing of Multi-Priority Traffic in Wireless Sensor Networks," Proc. 6th Annual Conference of International Technology Alliance, Southampton, UK, September 2012.

[10] Jenn-Yue Teo, Yajun Ha, Chen-Khong Tham, "InterferenceMinimized Multipath Routing with Congestion Control in Wireless Sensor Network for High-Rate Streaming," IEEE TRANSACTIONS ON MOBILE COMPUTING, vol. 7, no. 9, pp. 1124-1137, September 2008. Jenn-Yue Teo, Yajun Ha, ChenKhong Tham, "Interference-Minimized

[11] Ravindra Gupta, Hema Dhadhal, "Secure Multipath routing in Wireless Sensor Networks,” International Journal of Electronics and Computer Science Engineering, vol. 1, no. 2, pp. 585-589, August 2012. 